\title{
Erratum to: Vaccination with ErbB-2 peptides prevents cancer stem cell expansion and suppresses the development of spontaneous tumors in MMTV-PyMT transgenic mice
}

Eun-Young Gil ${ }^{1} \cdot$ Uk-Hyun Jo $^{1} \cdot$ Hye Jin Lee ${ }^{1} \cdot$ Jinho Kang $^{1} \cdot \mathrm{Jae} \mathrm{Hong} \mathrm{Seo}^{1}$. Eun Sook $\mathrm{Lee}^{2} \cdot$ Yeul Hong Kim ${ }^{1} \cdot \mathrm{InSun} \mathrm{Kim}^{3} \cdot$ Vy Phan-Lai ${ }^{4}$ Mary L. Disis ${ }^{5}$. Kyong Hwa Park ${ }^{1}$

Published online: 18 February 2016

(C) Springer Science+Business Media New York 2016

Erratum to: Breast Cancer Res Treat (2014)

147:69-80

DOI 10.1007/s10549-014-3086-4

In the original publication, the Fig. 2a was published incorrectly. The corrected Fig. 2a is given below:

The online version of the original article can be found under doi:10.1007/s10549-014-3086-4.

Kyong Hwa Park

khpark@korea.ac.kr

1 Division of Oncology/Hematology, Departments of Internal Medicine, Korea University Anam Hospital, Korea

University College of Medicine, 73 Inchon-ro, Seongbuk-Gu,

Seoul 136-705, Republic of Korea

2 Research Institute and Hospital, National Cancer Center, Goyang, Gyeonggi, Korea

3 Departments of Pathology, College of Medicine, Korea University, Seoul, Republic of Korea

4 Center for Global Mentoring, UCLA-DOE Institute, University of California Los Angeles, Los Angeles, CA, USA

5 Tumor Vaccine Group, University of Washington, Seattle, WA, USA 
A

\section{Immunization In vitro stimulation}

PBS
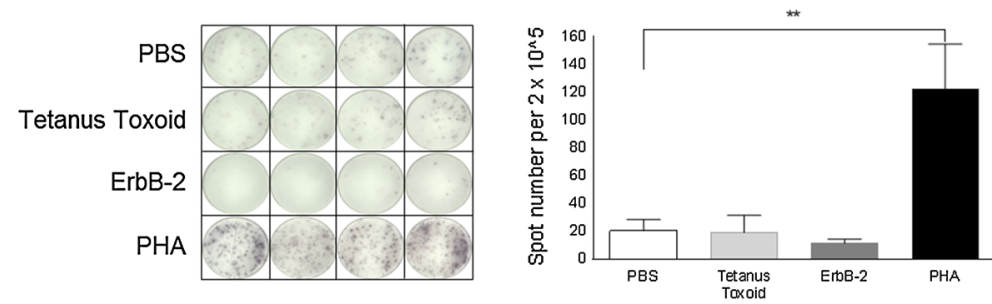

PBS
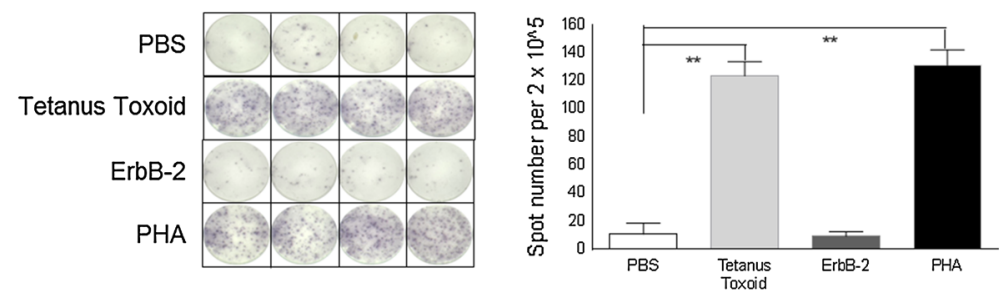

TT peptide
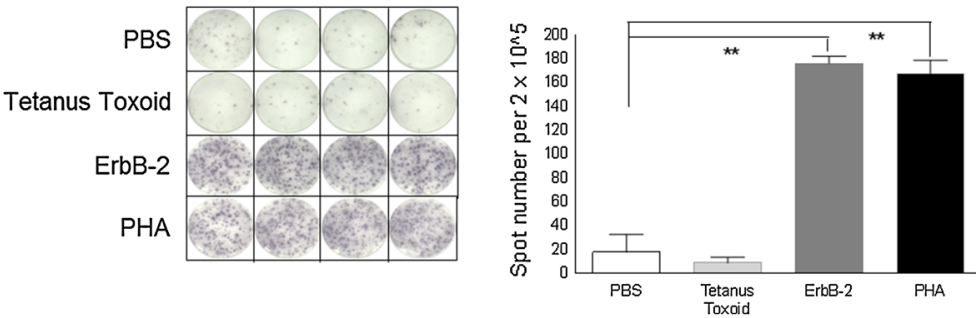

B

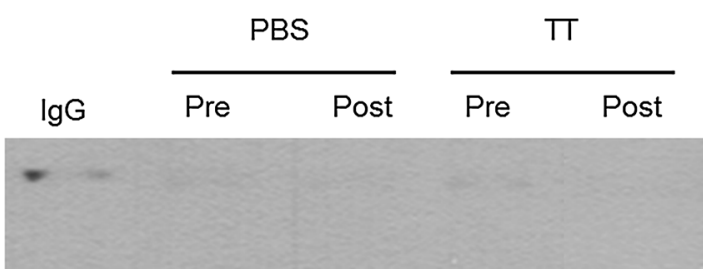

Fig. 2 ErbB-2-specific immunity was induced in tumor-free MMTVPyMT mice. a IFN-c ELISPOT using splenocytes from vaccinated mice. Columns, mean spots for six replicates; bars, SD. ${ }^{*} p<0.05$;
** $p<0.005$ versus spots obtained from PBS wells. b Immunoprecipitation with pre- and post-immunization sera from mice of each experimental group and lysates of MMC, a source of murine ErbB-2 